\title{
ASS kann das Krebsrisiko senken
}

Die regelmäßige Einnahme von Acetylsalicylsäure (ASS) senkt das Risiko, an Krebs zu erkranken. Der Effekt kommt vor allem durch die reduzierte Inzidenz von gastrointestinalen und hier besonders von kolorektalen Tumoren zustande. Diese Erkenntnisse gehen auf die Daten der Nurses' Health Study und der Health Professionals Follow-up Study zurück. Daran hatten sich über 135.000 Frauen und Männer beteiligt.

Während der 32-jährigen Nachbeobachtungszeit erkrankten knapp $21 \%$ der Studienteilnehmer an Krebs. Bei regelmäßiger ASS-Einnahme fiel die Krebsinzidenz insgesamt um $3 \%$ niedriger aus - eine geringe, aber signifikante Reduktion. $\mathrm{Zu}$ danken war der Effekt vor allem dem Rückgang von gastrointestinalen Malignomen mit einem Minus von $15 \%$. Speziell die Verminderung von kolorektalem Krebs um 19\% schlug hier zu Buche. Als regelmäßig galt eine mindestens zweimal wöchentliche Einnahme von ASS. Der Effekt war dosisabhängig, was für einen kausalen Effekt spricht, ohne ihn freilich zu beweisen. Zu beobachten war die protektive Wirkung ab einer Dosis von 162,5-487,5 mg ASS pro Woche, sofern sie sechs bis acht Jahre lang eingenommen wurde. Bei dieser Menge waren $14,0 \%$ weniger kolorektale Karzinome zu verzeichnen. Bei 15 oder mehr Tabletten à $325 \mathrm{mg}$ pro Woche lag die Reduktion bei $39,0 \%$.

Chan und Kollegen berechneten, dass der regelmäßige ASS-Konsum bei Personen über 50 Jahren 33 kolorektale Karzinome pro 100.000 Personenjahre verhinderte. Auch nach der Teilnahme am Koloskopiescreening auf Darmkrebs war ein positiver Effekt zu verzeichnen. Bei diesen Probanden wurden 18 Darmkrebsfälle pro 100.000 Personenjahre verhindert. Bezogen auf die Bevölkerung verhinderte der regelmäßige ASS-Kon-
Die regelmäßige Einnahme von ASS hat einen Zusatzeffekt: Sie reduziert das Krebsrisiko.

sum $1,8 \%$ sämtlicher, $8 \%$ der gastrointestinalen und $10,8 \%$ der kolorektalen bösartigen Tumoren. Mit Blick auf über 50-Jährige lag die Reduktion der Darmkrebsinzidenz bei $17,0 \%$, sofern die Betreffenden nicht am Koloskopiescreening teilnahmen, und bei $8,5 \%$, sofern sie sich dem Screening unterzogen.

Eine Assoziation des ASS-Konsums mit dem Risiko für Brust- bzw. Lungenkrebs oder für fortgeschrittenes Prostatakarzinom war nicht zu verzeichnen.

Robert Bublak

Cao Y et al. Population-wide Impact of Long-term Use of Aspirin and the Risk for Cancer. JAMA Oncol 2016; Mar 8 (Epub ahead of print).

\section{kurz notiert}

\section{Forschungsstandort Deutschland}

\section{Diagnose per Schwarmintelligenz +++} Die Genauigkeit von Hautkrebsdiagnosen kann verbessert werden, wenn die Bewertungen von mehreren Hautärzten zusammengeführt werden, fanden Berliner Forscher heraus. Bereits drei unabhängige ärztliche Meinungen erhöhen die Diagnosegenauigkeit gegenüber der Bewertung des besten Hautarztes. Mit steigender Anzahl der Bewertungen lässt sich die Genauigkeit sogar noch weiter erhöhen. Mehr als zehn Bewertungen bringen jedoch keinen Zusatznutzen [Kurvers RHJM et al. JAMA Dermatol. 2015; 151(12):1346-53]. red.

Krebsfrüherkennung im MRT +++

Cucurbituril kann den Kontrast von MRT-Aufnahmen etwa 100-fach besser verstärken als bisherige Kontrastmittel. Eine Bremer Arbeitsgruppe hat nun nachgewiesen, dass die LysinDecarboxylase eine Substanz produziert, die den Hohlraum des Cucurbituril-Rings besetzt und somit die im MRT messbaren Signale verändert. Gleichzeitig spielt das Enzym eine entscheidende Rolle beim Wachstum von Tumoren. Im Umkehrschluss konnten die Forscher aus den MRT-Signalen auf die Bösartigkeit eines Tumors schließen [Schnurr $M$ et al. Angew Chem. 2015;127(45):13645-8].

\section{Akute lymphoblastische Leukämie}

\section{Mutationen mit prognostischem Einfluss}

Bei Jugendlichen und Erwachsenen mit Precursor-B-Zell-ALL gehen Deletionen verschiedner Gene mit einer ungünstigen Prognose einher: darunter die von EBF1 („early B cell factor 1 “), IKZF1 („Ikaros family zinc finger protein $1^{\text {“) }}$ und CDKN2A/B („,cyclin-dependent kinase inhibitor 2A/B"). Zu diesem Ergebnis kam die PETHEMA-Gruppe (Spanish Programa Espanol de Tratamientos en Hematologia group), nachdem sie die Häufigkeit und den prognostische Einfluss von Veränderungen der Kopienzahl in zwölf Genregionen bei 142 Jugendlichen und Erwachsenen mit De-novoPrecursor-B-Zell-ALL untersucht hatte.

Eine Deletion des Gens für CDKN2A/B war die häufigste Veränderung der Kopienzahl (42\%), gefolgt von IKZF1-Deletionen $(35 \%)$. Letztere kamen häufiger bei Philadelphia-Chromosom (Ph)-positiver ALL als bei Ph-negativer ALL vor (52 vs. $30 \%)$. Außerdem waren IKZF1-Deletionen mit einem höheren Alter und höheren
Leukozytenzahlen assoziiert. Die multivariate Analyse ergab, dass höheres Alter und EBF1-Deletionen mit niedrigeren $\mathrm{Ra}$ ten an komplettem Therapieansprechen assoziiert waren (Hazard Ratios [HR] 0,949 bzw. 0,135; in der Ph-negativen Subgruppe: HR 0,946 bzw. 0,118). Hohe Leukozytenzahlen und fokale IKZF1-Deletionen korrelierten mit dem Rezidivrisiko (HR 1,005 bzw. 1,869). Höheres Alter und CDKN2A/B-Deletionen hatten einen negativen Einfluss auf das Gesamtüberleben (HR 1,038 bzw. 2,545; in der Ph-negativen Subgruppe: HR 1,044 bzw. 2,105).

Diese Ergebnisse weisen darauf hin, dass diese Veränderungen der Kopienzahl in die initiale Risikobeurteilung einfließen sollten.

Judith Neumaier

Ribera J et al. Prognostic Significance of Copy Number Alterations in Adolescent and Adult Patients With Precursor B Acute Lymphoblastic Leukemia Enrolled in PETHEMA Protocols. Cancer. 2015;121(21):3809-17. 